\title{
Fabrication and Characterization of Hybrid Tunnel Magnetoresistance Structures with Embedded Self-Assembled Nanoparticle Templates
}

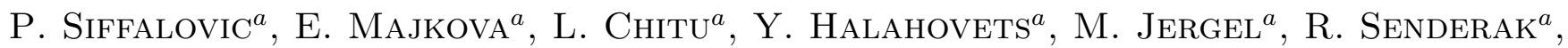

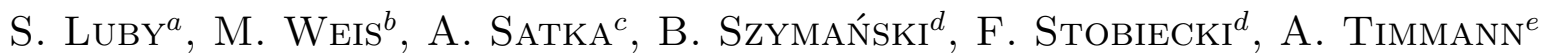 \\ AND S.V. Roth ${ }^{e}$ \\ ${ }^{a}$ Institute of Physics, Slovak Academy of Sciences \\ Dubravska cesta 9, 84511 Bratislava, Slovakia \\ ${ }^{b}$ Department of Physics, Slovak Technical University \\ Ilkovicova 3, 81219 Bratislava, Slovakia \\ ${ }^{c}$ International Laser Center, Ilkovicova 3, 81219 Bratislava, Slovakia \\ ${ }^{d}$ Institute of Molecular Physics, Polish Academy of Sciences \\ M. Smoluchowskiego 17, 60-179 Poznań, Poland \\ ${ }^{e}$ HASYLAB/DESY, Notkestr. 86, 22603 Hamburg, Germany
}

\begin{abstract}
We report on an incorporation of self-assembled templates of superparamagnetic $\mathrm{Fe}-\mathrm{O}$ nanoparticles into tunnel magnetoresistance devices. We fabricated a multilayer stack composed of the following layer sequence: $\mathrm{Cr} / \mathrm{Au} / \mathrm{Co} / \mathrm{NP} / \mathrm{Co} / \mathrm{Cu}$ on $\mathrm{Si}(100)$ substrate where NP stands for a self-assembled layer of nanoparticles deposited by the Langmuir-Blodgett technique. The X-ray reflectivity and grazing-incidence small angle X-ray scattering were employed to study the layers thicknesses and interface morphology in each preparation step. In particular, the grazing-incidence small angle X-ray scattering was measured before and after the nanoparticle incorporation as well as on the complete tunnel magnetoresistance stack. In this way, in-depth morphology profile during subsequent preparation steps was obtained. We demonstrate that X-ray analysis of the deposited tunnel magnetoresistance stack is essential for successful fabrication of novel hybrid devices consisting of self-assembled nanoparticles.
\end{abstract}

PACS numbers: 61.05.cf, 61.46.Df

\section{Introduction}

The progress in chemical synthesis of highly monodisperse superparamagnetic nanoparticles [1] opened new ways in applied spintronic research. Especially two-dimensional ordered layers of nanoparticles are very attractive as complementary to the deposited thin films. The self-assembling of nanoparticles into ordered templates can be realized by simple evaporation of the colloidal solution [2] or by more sophisticated LangmuirBlodgett (LB) deposition [3]. At the room temperature the magnetic moment of superparamagnetic nanoparticles is not pinned to crystalline lattice, thus allowing for efficient control by spin-polarized current. Here we report on the fabrication and structural characterization of hybrid tunnel magnetoresistance (TMR) devices with a tunnel barrier composed of two-dimensional layer of self-assembled $\mathrm{Fe}_{2} \mathrm{O}_{3}$ nanoparticles sandwiched between two Co layers. This novel structure will be employed in the second generation of current induced magnetization switching devices and spin torque nanooscillators [4].

\section{Experimental}

The structure $\mathrm{Cr}(5 \mathrm{~nm}) / \mathrm{Au}(40 \mathrm{~nm}) / \mathrm{Co}(20 \mathrm{~nm}) / \mathrm{NP} /$ $\mathrm{Co}(3 \mathrm{~nm}) / \mathrm{Cu}(3 \mathrm{~nm})$ was deposited on $\mathrm{Si}(100)$ wafer with native oxide layer, where NP stands for self-assembled monolayer of nanoparticles. In the first phase the $\mathrm{Cr}$ buffer layer, bottom Au electrode and Co layer were deposited in ultrahigh vacuum (UHV) chamber by means of e-beam evaporation. The evaporation was then interrupted and the sample was transferred for subsequent LB deposition. The natural thin Co oxide layer prevents the further stack oxidation. The synthesis of $\mathrm{Fe}_{2} \mathrm{O}_{3}$ nanoparticles stabilized by surfactant was described elsewhere [5]. The nanoparticle diameter is $6.4 \pm 0.6 \mathrm{~nm}$ as obtained by scanning electron microscopy (SEM) [2]. The nanoparticles are superparamagnetic with $22 \mathrm{~K}$ blocking temperature. The diluted colloidal nanoparticle solution was dropwisely applied on water surface in LB trough. In the second phase the nanoparticle monolayer was deposited onto substrate by a single dip at $15 \mathrm{mN} / \mathrm{m}$ surface pressure. In the third phase after monolayer depo- 
sition the sample was transferred again into the UHV chamber and the top Co layer and $\mathrm{Cu}$ cap layer were deposited. The grazing-incidence small angle X-ray scattering (GISAXS) at BW4 beamline of Hamburger synchrotron was employed to measure X-ray diffuse scattering pattern by two-dimensional detector [6]. The X-ray wavelength was set to $0.138 \mathrm{~nm}$ and sample-detector distance was $225 \mathrm{~cm}$. The angle of incidence was set to $0.7^{\circ}$. The X-ray reflectivity (XRR) was measured at X-ray diffractometer (Bruker D8 Discover) equipped with an $18 \mathrm{~kW} \mathrm{Cu}$ rotating anode (wavelength of $0.154 \mathrm{~nm}$ ) and the Goebel mirror.

\section{Results and discussion}

After first deposition step the as-prepared substrate was characterized by XRR and GISAXS. The measured XRR curve as well as simulation is shown in Fig. 1a. A genetic fitting algorithm and reflectivity calculation based on the Parratt formalism with a Nevot-Croce interface roughness model was used to estimate the individual thickness and roughness of each layer. The fit results provided the stack: $\mathrm{Si}$ (substrate $\| 0.3) / \mathrm{SiO}_{2}(1.8 \| 0.3) /$ $\mathrm{Cr}(8.2 \| 0.8) / \mathrm{Au}(38.3 \| 0.8) / \mathrm{Co}(23.5 \| 1.5) / \mathrm{Co}_{2} \mathrm{O}_{3}(2.2 \| 1.1)$ where the film thickness and roughness, respectively, are given in nanometers. The reflectivity measurement provides root-mean-square (rms) roughness of each layer in the stack. The non-coplanar X-ray scattering adds information on the autocorrelation and cross-correlation height functions of interfaces. The measured GISAXS pattern is shown in Fig. 1b. The regularly spaced streaks
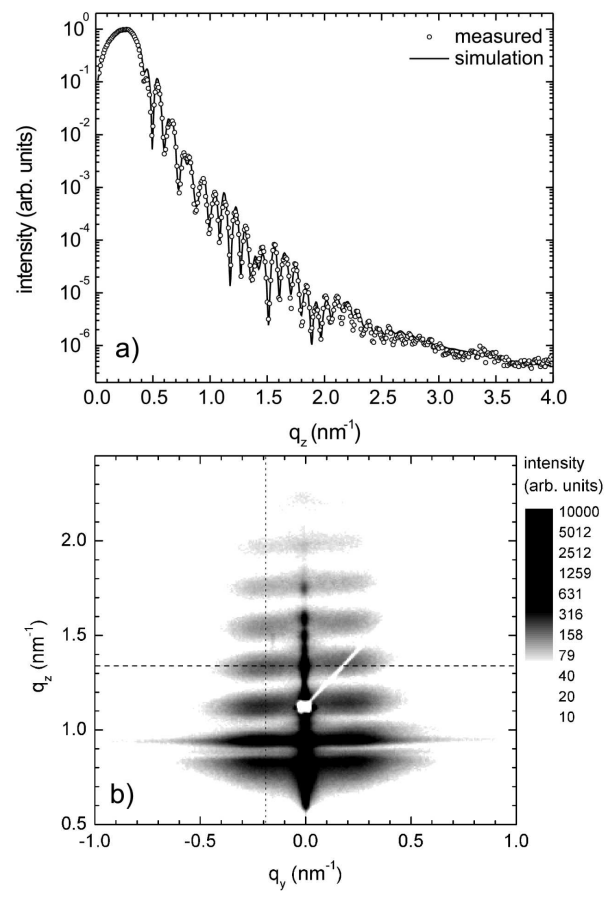

Fig. 1. (a) The measured and simulated XRR curves. (b) The measured GISAXS pattern. of diffusely scattered radiation, also known as resonant diffuse scattering (RDS) [7], arise as a consequence of vertically correlated interface roughness. The maxima along $q_{z}$ direction are attributed to the constructive interference of the partially scattered radiation by each interface in the multilayer stack. The first two maxima, also known as the Yoneda peaks, are corresponding to the scattered radiation exiting at the critical angle for $\mathrm{Co}$ and $\mathrm{Au}$ layer. The second Yoneda peak, at approximately $q_{z}=0.95 \mathrm{~nm}^{-1}$, related to the Au layer is dynamically enhanced in intensity due to the overlapping RDS peak. Figure 2a shows a line-cut and its integral at $q_{z}=1.33 \pm 0.02 \mathrm{~nm}^{-1}$ (indicated with a dashed line in Fig. 1b) extracted from the GISAXS pattern. At this relatively large $q_{z}$ value the multiple scattering events confined to the critical exit angle can be neglected and the simple Born approximation (BA) [7] can be used for the results interpretation. The RDS intensity along $q_{y}$ at constant $q_{z}$ is in the first approximation given by the summation of all Fourier transformed cross-correlation functions of interfaces multiplied by the respective phase factors responsible for the existence of maxima along $q_{z}$ direction [7]. In the case of interfaces with identical autocorrelation functions and within the small roughness approximation $\left(q_{z}^{2} \sigma^{2} \ll 1\right)$ the measured line-cut will be proportional to the power spectral density (PSD) function of interface roughness. The measured curve shows slightly peaked plateau at $q_{y} \approx 0.19 \mathrm{~nm}^{-1}$ which is visible in all RDS sheets. Its position is denoted by dotted line in Fig. 1b. Under the assumption of identical or at least similar morphology of interfaces the measured curve would represent the PSD of interface roughness with the typical lateral correlation length $\Delta_{y}=2 \pi / q_{y} \approx 33 \mathrm{~nm}$ which is compatible with the cluster growth of metallic thin films especially of Au. The simulation of the RDS along $q_{y}$ was modeled by BA taking into account the interface reflectivity calculated within the dynamical theory of reflection. For each interface we used the autocorrelation function $\sigma^{2} \exp \left(-(R / \xi)^{2 H}\right)$ proposed by Sinha [7], with lateral correlation length $\xi=30 \mathrm{~nm}$, the Hurst parameter $H=0.8$ and interface roughness $\sigma$. Roughness replication was implemented by the Ming model with vertical correlation length $\Lambda=50 \mathrm{~nm}$. The simulated curve is shown as a dashed line in Fig. 2a. There is a clear discrepancy between the measured data and simulation. The used interface autocorrelation is monotonously decreasing function and cannot account for the existence of clusters on metal surfaces. On the other hand, the integrals (Fig. 2a) under the measured and simulated curves are converging in the limit of high $q_{y}$ values. Because the rms interface roughness is proportional to the square root of the integral of PSD function, this proves that the rms interface roughness determined by XRR is correct. The observed substrate roughness does not affect the transfer quality of the self-assembled monolayer of nanoparticles. The SEM image of the surface after LB deposition is shown in Fig. 2b. The long-range order in the nanoparticle monolayer is precluded by the nanopar- 

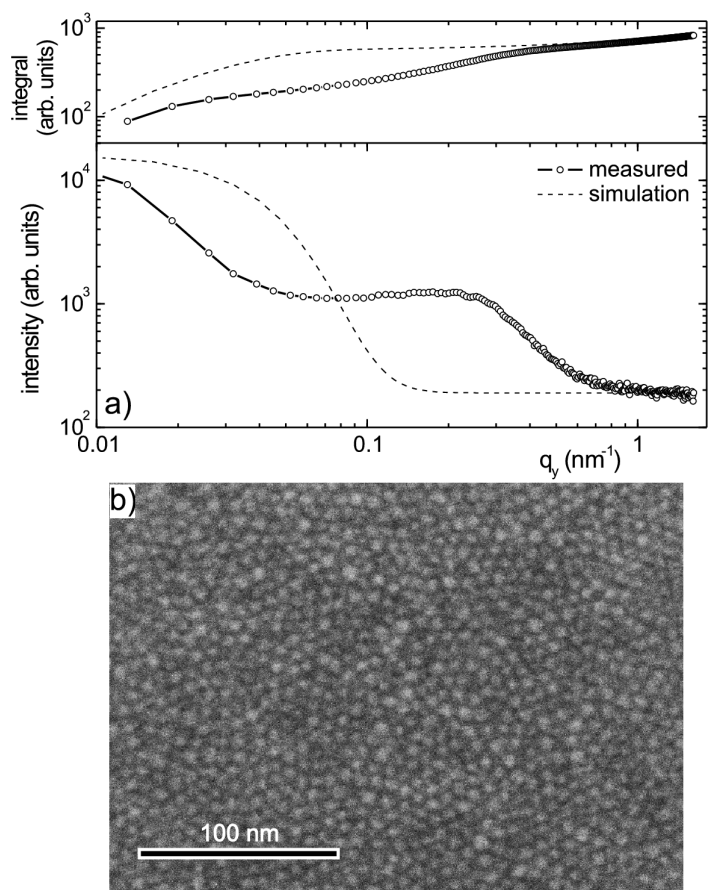

Fig. 2. (a) The simulated and measured line profiles and their integrals at $q_{z}=1.33 \mathrm{~nm}^{-1}$. (b) A SEM micrograph of self-assembled $\mathrm{Fe}_{2} \mathrm{O}_{3}$ nanoparticle monolayer.

ticle size distribution. The global probe of the nanoparticle arrangement is provided by GISAXS measurement. Figure $3 \mathrm{a}$ shows the measured GISAXS pattern. The side maxima at $\left|q_{y}\right| \approx 0.83 \mathrm{~nm}^{-1}$ are an evidence for the self-assembled monolayer. The average nanoparticle spacing is approximately $\Delta_{y}=2 \pi / q_{y} \approx 7.6 \mathrm{~nm}$. To obtain more quantitative comparison of samples with the self-assembled nanoparticles covered with different thicknesses of top $\mathrm{Co}$ layer and $\mathrm{Cu}$ cap layer we integrated the line-cuts for $q_{z} \in\langle 0.67,0.86\rangle \mathrm{nm}^{-1}$ (interval between the two dashed lines in Fig. 3a). Figure $3 \mathrm{~b}$ shows the integrated line-cuts for bare nanoparticles and nanoparticles covered with various thicknesses of Co top layer and $\mathrm{Cu}$ cap layer. The effect of growing Co layer thickness is visible as a broadening of the central peak at $q_{y}=0$ and decreasing intensity of the satellite peaks at $\left|q_{y}\right| \approx 0.83 \mathrm{~nm}^{-1}$ corresponding to the self-assembled monolayer of nanoparticles. The broadening of central peak comes from the partially replicated nanoparticle roughness of the growing top Co layer which produces significant diffuse scattering. The signal at side maxima is overlaid by monotonous diffuse scattering from the growing top Co layer which also suppresses by absorption GISAXS originating from underlying self-assembled nanoparticles. Deposition of thin $\mathrm{Cu}$ cap layer results in further suppression of the diffusely scattered radiation stemming from the nanoparticle template. Regardless top Co layer and $\mathrm{Cu}$ cap layer the measured curve still reveals the underlying self-assembled nanoparticle mono-
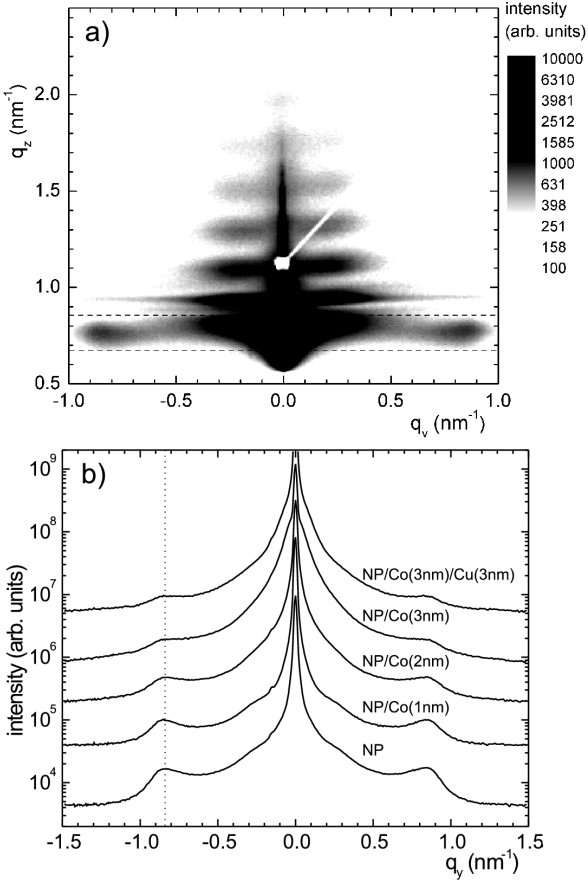

Fig. 3. (a) A GISAXS pattern of self-assembled monolayer. (b) A set of curves showing the effect of deposited layers.

layer and serves as non-destructive probe of the buried nanoparticles.

In summary, we have fabricated hybrid TMR structure with tunnel barrier composed of self-assembled superparamagnetic nanoparticles. The structure and morphology of the bottom stack $\mathrm{Cr} / \mathrm{Au} / \mathrm{Co} / \mathrm{Co}_{2} \mathrm{O}_{3}$ serving as substrate has been studied in detail. The self-assembled layer of nanoparticle was deposited by LB method and verified by SEM and GISAXS technique. The overgrowing Co layer was monitored by GISAXS. The diffusely scattered radiation originating from the ordered nanoparticle template is detectable even for $3 \mathrm{~nm}$ thick layers of $\mathrm{Co}$ and $\mathrm{Cu}$ grown on nanoparticle template. The GISAXS offers a non-destructive probe of ordered nanoparticle monolayers in hybrid spintronic devices.

\section{Acknowledgments}

The work was supported by VEGA grant no. $2 / 6030 / 26$, and $2 / 0047 / 08$, by Centre of Excellence SAS contract no. I/2/2008 and by APVV grant No. LPP0080-06 and 0173-06. The supports of EU HASYLAB grant RII3-CT-2004-506008, MNT-ERA-2007-009SK and NATO CLG 982748 grant are also acknowledged.

\section{References}

[1] J. Park, K. An, Y. Hwang, J.G. Park, H.J. Noh, J.Y. Kim, J.H. Park, H.M. Hwang, T. Hyeon, Nature Mater. 3, 891 (2004). 
[2] P. Siffalovic, E. Majkova, L. Chitu, M. Jergel, S. Luby, A. Satka, S.V. Roth, Phys. Rev. B 76, 195432 (2007).

[3] T. Fried, G. Shemer, G. Markovich, Adv. Mater. 13, 1158 (2001).

[4] M. Tsoi, Nature Phys. 4, 17 (2008).

[5] S. Sun, H. Zeng, D.B. Robinson, S. Raoux, P.M. Rice, S.X. Wang, G. Li, J. Am. Chem. Soc. 126, 273 (2004).
[6] S.V. Roth, R. Döhrmann, M. Dommach, M. Kuhlmann, I. Kröger, R. Gehrke, H. Walter, C. Schroer, B. Lengeler, P. Müller-Buschbaum, Rev. Sci. Instrum. 77, 085106 (2006).

[7] V. Holý, U. Pietsch, T. Baumbach, High-Resolution $X$-Ray Scattering from the Thin Films and Multilayers, Springer, Berlin 1999. 